TRANSACTIONS OF THE

AMERICAN MATHEMATICAL SOCIETY

Volume 355, Number 11, Pages 4349-4363

$\mathrm{S} 0002-9947(03) 03160-\mathrm{X}$

Article electronically published on July 2, 2003

\title{
RIGIDITY IN HOLOMORPHIC AND QUASIREGULAR DYNAMICS
}

\author{
GAVEN J. MARTIN AND VOLKER MAYER
}

\begin{abstract}
We consider rigidity phenomena for holomorphic functions and then more generally for uniformly quasiregular maps.
\end{abstract}

\section{INTRODUCTION}

1.1. Rigidity of rational functions. Fatou's problem of the density of hyperbolic maps in the space of rational functions is one of the major problems in the field of holomorphic dynamics. It has been reduced by Mañé, Sad and Sullivan [13] to the no invariant lines field (NILF) conjecture. A rational map $f$ has an invariant line field if the Julia set has positive Lebesgue measure, $\left|\mathcal{J}_{f}\right|>0$, and if there is a measurable map $z \in \mathcal{J}_{f} \mapsto \mathcal{L}_{z} \in[0, \pi)$ with

$$
\mathcal{L}_{f(z)}=\mathcal{L}_{z}+\arg f^{\prime}(z) \bmod \pi .
$$

It is well known that the map $f$ has an invariant line field precisely when $f$ admits a quasiconformal deformation that is non-trivial on the Julia set. Now, the NILF-conjecture suggests that a rational function does not admit an invariant line field unless it is a map of a very special kind, that is, a Lattès map. An affirmative answer to this conjecture implies the density of hyperbolic maps [13. We recall that a Lattès map is a rational function $f$ that is obtained from semi-conjugating an expanding similarity $A(z)=\lambda z,|\lambda|>1$, by a certain automorphic meromorphic function $h: \mathbb{C} \rightarrow \hat{\mathbb{C}}$. Such maps $f$ are then obtained as solutions of the functional equation

$$
f \circ h(z)=h \circ A(z), \quad z \in \mathbb{C} .
$$

Next, from Zalcman's normality criteria [30, 27] we know that each point $z_{0}$ of the Julia set $\mathcal{J}_{f}$ of $f$ admits a renormalization of the following form. There are a sequence of points $z_{j} \rightarrow x_{0}$ and positive real numbers $\rho_{j} \searrow 0$ and a subsequence of iterates $f^{k_{j}}$ such that

$$
f^{k_{j}}\left(\rho_{j} z+z_{j}\right) \rightarrow \Psi(z), \quad z \in \mathbb{C},
$$

the convergence is locally uniform in $\mathbb{C}$ and $\Psi: \mathbb{C} \rightarrow \hat{\mathbb{C}}$ is a non-constant meromorphic limit function.

Received by the editors October 19, 1999.

2000 Mathematics Subject Classification. Primary 30C65; Secondary 37F45.

This research was partially supported by a grant from the Marsden Fund (NZ).

(C)2003 American Mathematical Society 
Following an analogy with the limit sets of Kleinian groups, we call a point $z_{0} \in \mathcal{J}_{f}$ a conical point if the sequence above in (2) can be chosen to have the additional property that there is a constant $M<\infty$ such that

$$
\frac{\left|z_{0}-z_{j}\right|}{\rho_{j}}<M
$$

and we require only that the convergence be uniform on $\mathbb{D}$.

The set of conical points is denoted $\Lambda_{c}$. A typical conical point is a repelling fixed point. In general $\Lambda_{c}$ is a large subset of the Julia set, and in many cases, for example the Lattès maps, we even have $\Lambda_{c}=\mathcal{J}_{f}$. Reinforcing the analogy with Kleinian groups, we shall see that examples of points in the Julia set which are not conical are the parabolic fixed points (see Lemma 3.5.).

Several other notions of conical points appear in the literature, and we discuss these later in $\S 3$.

The following lemma gives a characterisation of a conical limit point which is easier to work with.

Lemma 1.1. Let $z_{0} \in \mathcal{J}_{f}$ be a conical point for the mapping $f$. Then there are a sequence $\rho_{j} \searrow 0$, an increasing sequence of natural numbers $\left\{k_{j}\right\}_{j=1}^{\infty}$ and a nonconstant meromorphic function $\Psi$ such that

$$
f^{k_{j}}\left(z_{0}+\rho_{j} z\right) \rightarrow \Psi
$$

uniformly in $\mathbb{D}$.

Proof. Let $\rho_{j}, z_{j}, k_{j}, \Psi$ and $M$ be given as in the definition of a conical limit point. Set $\phi_{j}(z)=\rho_{j} z+z_{j}$ and $\varphi_{j}(z)=\rho_{j} z+z_{0}$. Then

$$
f^{k_{j}} \circ \varphi_{j}(z)=f^{k_{j}} \circ \phi_{j}(z) \circ \phi_{j}^{-1}(z) \circ \varphi_{j}(z) .
$$

The result now follows after passing to a subsequence, since $f^{k_{j}} \circ \phi_{j}(z) \rightarrow \Phi$ and the sequence $\phi_{j}^{-1}(z) \circ \varphi_{j}(z)=z+\left(z_{0}-z_{j}\right) / \rho_{j}$ is precompact.

We may reformulate the NILF-conjecture, replacing role of the Julia set by $\Lambda_{c}$. We shall prove the following result:

Theorem 1.2. Suppose the conical set of the rational function $f$ has positive Lebesgue measure, $\left|\Lambda_{c}\right|>0$. Then either $f$ is a Lattès map or $f$ has no invariant line field.

Note that the conical set has either zero or full measure. Therefore the condition $\left|\Lambda_{c}\right|>0$ implies in particular that $\mathcal{J}_{f}=\hat{\mathbb{C}}$. In view of the above result it would be interesting to know an example of a rational function for which the Julia set has positive measure and the conical set has zero measure. Such a phenomena appears in the space of entire functions. McMullen [19] showed that $f(z)=\sin z$ has a Julia set of positive measure and, since this function has a non-empty Fatou set, its conical set has zero measure.

Theorem 1.2 is not really new (compare 20, [12, [6]). However, we give a new and elementary proof of this fact that in particular does not involve any orbifold theory. We use the possibility of renormalization at conical points to construct explicitly the necessary automorphic function of the equation (1.1). A similar idea has been used by Berteloot and Loeb in [1, where they gave a geometric description of the Lattès maps. The proof we give also has the advantage that it does not use that $f$ is a global holomorphic map of $\hat{\mathbb{C}}$. Therefore, Theorem 1.2 is also true for 
entire functions. One of our primary motivations in looking for such a proof is its applicability to higher-dimensional analogues of holomorphic dynamics, uniformly quasiregular maps, which we now present.

1.2. Rigidity in higher dimensions. The maps we shall consider in the sequel are self-maps of the $n$-sphere $\overline{\mathbb{R}}^{n}=\mathbb{R}^{n} \cup\{\infty\}, n \geq 2$, that preserve a conformal structure. Such a structure is a bounded measurable map $\mu: \overline{\mathbb{R}}^{n} \rightarrow S(n)$, the space of symmetric positive definite $n \times n$ matrices of determinant 1 . More precisely, let $f$ be a map that is in the Sobolev class $W^{1, n}\left(\overline{\mathbb{R}}^{n}, \overline{\mathbb{R}}^{n}\right)$ whose Jacobian determinant $\operatorname{det} f^{\prime}>0$ almost everywhere. The pull-back action of $f$ on a conformal structure $\mu$ is given by

$$
f^{*} \mu(x)=\operatorname{det}\left(f^{\prime}(x)^{t}\right)^{\frac{-2}{n}} f^{\prime}(x) \mu(f(x)) f^{\prime}(x) \quad \text { for } \quad \text { a.e. } x \in \overline{\mathbb{R}}^{n} .
$$

We call $f \mu$-rational if $\mu$ is $f$-invariant, meaning

$$
f^{*} \mu=\mu \quad \text { a.e. }
$$

This equation has the following geometric interpretation: Consider the field of $\mu^{-}$ spheres $\Sigma_{x, r}^{\mu}=\left\{v \in \mathbb{R}^{n} ;{ }^{t} v \mu(x) v=r\right\}$, which is in fact a measurable Euclidean ellipsoid field on $\overline{\mathbb{R}}^{n}$ such that the eccentricity of the ellipsoids is uniformly bounded. Now, a $\mu$-rational map is conformal in this structure in the sense that it preserves this field of $\mu$-spheres.

A $\mu$-rational map is quasiregular (see $\S 2$ or [26] for appropriate definitions), and the dilatation of any $\mu$-rational map only depends on the eccentricity of the ellipsoid field $\Sigma_{x, r}^{\mu}$. Therefore, for a given conformal structure $\mu$ there is a fixed constant $K \geq 1$ such that any $\mu$-rational map is $K$-quasiregular. In particular, if $f$ is $\mu$-rational, then the family of all the iterates $f^{\ell}$ has a uniform dilatation bound. Such maps are called uniformly quasiregular, or uqr for brevity. Conversely, it is known that any uqr map is $\mu$-rational with respect to some conformal structure $\mu$ [9].

In two dimensions the study of uqr maps reduces to the classical case of (holomorphic) rational functions. In fact, when $\mu \equiv I d$ is the standard Euclidean structure, then (1.7) is nothing other than the usual Cauchy-Riemann system. If $n=2$, the $I d$-rational maps are precisely the holomorphic functions of $\hat{\mathbb{C}}$, and hence they are rational maps. Moreover, from the Ahlfors-Bers measurable Riemannian mapping theorem (the existence theorem for quasiconformal mappings) it follows that any uqr map in two dimensions is quasiconformally conjugate to a rational map. Hence we restrict our investigation in two dimensions to the rational functions. The picture is completely different for $n \geq 3$. From Liouville's theorem we find that the only $I d$-rational mappings are Möbius transformations. The only way to produce non-injective uqr maps is to allow some distortion and very little regularity of the conformal structure. Thus we consider only measurable conformal structures. Nevertheless, it is a difficult task to write down explicit examples of uqr mappings having degree at least two. This was first done by Iwaniec and Martin [9, and subsequently other examples were given in [14, [17], [18. In particular, Mayer's paper 17] exhibits the higher-dimensional Lattès maps. They are, as before, defined by the functional equation (1.1), where this time $h: \mathbb{R}^{n} \rightarrow \overline{\mathbb{R}}^{n}$ is a quasimeromorphic map which is automorphic with respect to some crystallographic group.

The rigidity result we present below shows that there are in fact far fewer uqr maps with a large Julia set than there are rational functions. We first explain how 
this is related to Theorem 1.2 A quasiconfomal deformation of a rational map $f$ is again a rational function $g$ with $g=\varphi^{-1} \circ f \circ \varphi$ and $\varphi$ a quasiconformal mapping that is not a Möbius transformation. Let us say that such a deformation is non-trivial on the Julia set if the Lebesgue measure of the Julia set $\left|\mathcal{J}_{f}\right|>0$ and if the restriction of $\varphi$ to $\mathcal{J}_{f}$ is not the restiction of a holomorphic map. Note that such a conjugacy induces an $f$-invariant conformal structure $\mu=\varphi^{*} I d$, and, conversely, it follows from the Ahlfors-Bers theorem that a rational map that preserves a conformal structure can be quasiconformally deformed. Since we are deforming holomorphic functions, one can replace in this discussion the conformal structures by simpler objects such as the invariant line fields (for instance the line field generated by the major axis of the ellipse field that is induced by an invariant conformal structure). Thus we can reformulate Theorem 1.2 in the following way:

If $f$ is a rational function with $\left|\Lambda_{c}\right|>0$, then either $f$ is a Lattès map, or the standard structure Id is the unique $f$-invariant conformal structure.

When the dimension $n \geq 3$, this second alternative cannot occur, since an $I d$-rational map is a Möbius transformation. Hence the following result is the precise higher-dimensional analogue of Theorem 1.2. One might compare this with the classical Mostow rigidity theorem.

Theorem 1.3. Either the set of conical points $\Lambda_{c}$ of a map $f \in U Q R\left(\overline{\mathbb{R}}^{n}\right), n \geq 3$, has zero measure, or $f$ is a chaotic Lattès map (and then $\Lambda_{c}=\overline{\mathbb{R}}^{n}$ ).

This higher-dimensional rigidity result suggests that the NILF-conjecture has the following generalization:

Conjecture 1.4. Suppose $f \in U Q R\left(\overline{\mathbb{R}}^{n}\right), n \geq 3$, with a Julia set of positive measure (or possibly $\mathcal{J}_{f}=\overline{\mathbb{R}}^{n}$ ). Then $f$ is a Lattès map.

Note that the Lattès examples are quite rare in the space of all rational functions and even in the subspace of those functions that have a conical set of positive measure. Here are two examples.

Example 1.5. The rational functions

$$
f_{p}(z)=\left(1-\frac{2}{z}\right)^{2 p}, \quad p=1,2, \ldots,
$$

are all semi-hyperbolic with $\mathcal{J}_{f_{p}}=\Lambda_{c}=\hat{\mathbb{C}}$. But the only Lattès map of this family corresponds to the value $p=1$. Therefore, only $f_{1}$ has a higher-dimensional uqr analogue.

The degree of the above functions does increase with $p$. But we may fix this degree and see the same phenomenon from Lyubich's family

$$
g_{\lambda}(z)=1-\frac{\lambda}{z^{2}}, \quad \lambda \in \mathbb{C}^{*} .
$$

The parameter space $\mathbb{C}^{*}$ contains a subset Irr of unstable values (see [11] for details). There is only one parameter $\lambda=2 \in \operatorname{Irr}$ that corresponds to a Lattès map. On the other hand, a dense subset of $\operatorname{Irr}$ gives rise to strictly critically finite maps which have $\Lambda_{c}=\hat{\mathbb{C}}$. So, all these values of the big space $\operatorname{Irr}$ (which is in fact a set of positive measure, as Rees [25] has shown) reduce to one point $\lambda=2$ in higher dimensions. 
However, the aforementioned uqr map in [9] is not obtained by a Lattès construction, and the space $U Q R\left(\overline{\mathbb{R}}^{n}\right)$ may well contain many other examples with Cantor or other small Julia sets.

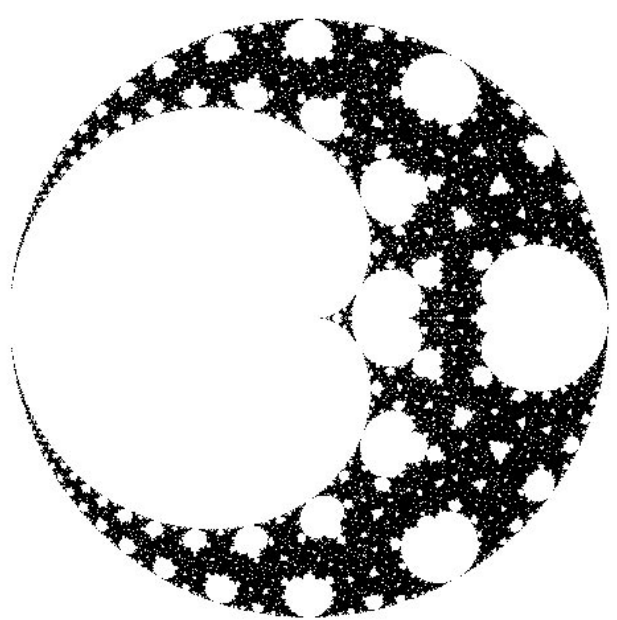

FiguRE 1. The set $\mathrm{Irr}$

\section{Quasiregularity and CONFORMal STRUCTUReS}

In this section we shall recall some of the necessary facts concerning uqr maps and conformal structures. The notation we use is quite standard. $\mathbb{B}^{n}$ denotes the unit ball of $\mathbb{R}^{n}$ and $\mathbb{S}^{n-1}$ its boundary. In two dimensions we often use complex notation and write for example $\mathbb{D}=\mathbb{B}^{2}$.

2.1. Uniformly quasiregular mappings. Let $D \subset \overline{\mathbb{R}}^{n}$ be a domain and $f: D \rightarrow$ $\overline{\mathbb{R}}^{n}$ a mapping of Sobolev class $W_{l o c}^{1, n}\left(D, \overline{\mathbb{R}}^{n}\right)$. We shall only consider orientationpreserving mappings; thus $\operatorname{det} f^{\prime}(x)>0$ for a.e. $x \in D$. Such a mapping is said to be $K$-quasiregular, where $1 \leq K<\infty$, if

$$
\max _{|h|=1}\left|f^{\prime}(x) h\right| \leq K \min _{|h|=1}\left|f^{\prime}(x) h\right| \text { for a.e. } x \in D .
$$

The smallest number $K$ for which the above inequality holds is called the dilatation of $f$. A non-constant quasiregular mapping can be redefined on a set of measure zero so as to make it continuous, open and discrete, and we shall always assume this to be the case. If $D$ is a domain of the compactification $\overline{\mathbb{R}}^{n}$ (equipped with the spherical metric; thus $\overline{\mathbb{R}}^{n}$ is isometric via stereographic projection with the $n$-sphere $\left.\mathbb{S}^{n}\right)$, then we use the chart at infinity $x \mapsto x /|x|^{2}$ to extend the notion of quasiregularity to mappings $f: D \rightarrow \overline{\mathbb{R}}^{n}$ in the obvious manner. Such mappings are also said to be quasimeromorphic . A mapping $f$ of a domain $D$ into itself is called uniformly quasiregular (uqr) if there is some $1 \leq K<\infty$ such that all the iterates $f^{k}$ are K-quasiregular. We abbreviate this to $f \in U Q R(D)$. 
The branch set $B_{f}$ is the set of points $x \in D$ for which $f$ is not locally homeomorphic at $x$. In the setting of uqr mappings there are analogues of the Picard and Montel theorems due to Rickman [26]. An easy application is that the exceptional set $\mathcal{E}_{f}$ of a $K$-uqr map $f$, the set of points that have only a finite number of images and preimages, contains at most a finite number $q=q(K, n)$ of points. Also we have the usual property

$$
\bigcup_{k \geq 0} f^{k}(\Omega) \supset \overline{\mathbb{R}}^{n} \backslash \mathcal{E}_{f}
$$

for every open set $\Omega$ that intersects the Julia set (see [15]).

The other important normality criterion is the following quasiregular version of Zalcman's lemma. It is due to Miniowitz [23].

Zalcman's Lemma. A family $\mathcal{F}$ of $K$-quasiregular mappings $f: \mathbb{B}^{n} \rightarrow \overline{\mathbb{R}}^{n}$ is not normal at $x_{0}$ if and only if there are positive numbers $\rho_{j} \rightarrow 0$, points $x_{j} \rightarrow x_{0}$ and mappings $f_{j} \in \mathcal{F}$ such that

$$
f_{j}\left(x_{j}+\rho_{j} x\right) \rightarrow \Psi(x)
$$

spherically uniformly on compact subsets of $\mathbb{R}^{n}$, where $\Psi: \mathbb{R}^{n} \rightarrow \overline{\mathbb{R}}^{n}$ is a nonconstant quasimeromorphic map.

In fact Miniowitz's proof does not include the condition $x_{j} \rightarrow x_{0}$. However, it can easily be achieved with slight modifications.

2.2. Conformal structures. Let $\mu: \overline{\mathbb{R}}^{n} \rightarrow S(n)$ be a measurable conformal structure as defined in $\S 1$. If we forget for a moment the boundedness and determinant 1 condition, then $\mu$ can be viewed as a standard Riemannian metric with measurable coefficients. Since the notion of conformality does not change if we rescale the metric, this explains the canonical normalisation to require that $\operatorname{det} \mu(x)=1$ for a.e. $x \in \overline{\mathbb{R}}^{n}$. The boundedness condition is necessary in order to retain the structures that are adapted to quasiregular mappings. It implies that there is a constant $K \geq 1$ such that

$$
\frac{1}{K}|X|^{2} \leq{ }^{t} X \mu(x) X \leq K|X|^{2} \quad \text { for all } X \in \mathbb{R}^{n} \text { and a.e. } x \in \overline{\mathbb{R}}^{n} \text {. }
$$

As we explained in $\S 1$, a conformal structure should really be viewed as a measurable field of ellipsoids of bounded eccentricity.

Recall the pull-back of a conformal structure $\mu$ by a quasiregular mapping $f$ :

$$
f^{*} \mu(x)=f^{\prime}(x)[\mu \circ f(x)]:=\frac{{ }^{t} f^{\prime}(x) \mu(f(x)) f^{\prime}(x)}{\operatorname{det} f^{\prime}(x)^{\frac{2}{n}}} \text { for a.e. } x \in \overline{\mathbb{R}}^{n},
$$

and note that this pull-back behaves nicely under composition:

$$
\left(f_{1} \circ f_{2}\right)^{*} \mu=f_{2}^{*}\left(f_{1}^{*} \mu\right) \text {. }
$$

Consequently, if $f$ is $\mu$-rational $\left(\mu(x)=f^{*} \mu(x)\right.$, a.e.), then it is automatically uniformly quasiregular. The converse is

Theorem ([9]). A mapping of the Sobolev class $W^{1, n}\left(\overline{\mathbb{R}}^{n}, \overline{\mathbb{R}}^{n}\right)$ is uniformly quasiregular if and only if it is $\mu$-rational for some measurable conformal structure $\mu$. 
For completeness we recall the following theorem in the form we shall use it.

Liouville's Theorem. Let $f: U \rightarrow V$ be quasiregular with $U, V$ domains of $\mathbb{R}^{n}$ and $n \geq 3$. If $f^{*} I d=I d$, then $f$ is the restriction of a Möbius transformation.

We have seen that in the context of quasiregular mappings there are powerful normality criteria. Recall too that if a sequence $\left\{f_{j}\right\}$ of $K$-quasiregular mappings converges to a mapping $f$, then $f$ is either constant or a quasiregular mapping. It will be important in our applications to know whether or not the conformal structures $\mu_{f_{j}}=f_{j}^{*} I d$ also converge. In general this is not the case (see [10] IV, $5.4])$.

In order to make clear the notion of convergence in the space of matrices $S(n)$ we recall now how the metric $\delta$ is defined in that space (see Helgason [7] for details). First of all, the general linear group $G l(n, \mathbb{R})$ acts on $S(n)$ via the rule

$$
A \mapsto X[A]=|\operatorname{det} X|^{-\frac{2}{n}^{t}} X A X, \quad X \in G l(n, \mathbb{R}), \quad A \in S(n),
$$

and the metric $\delta$ is invariant under this action. In particular,

$$
\delta(A, B)=\delta\left(\sqrt{B}^{-1}[A], I d\right)=\delta\left(\sqrt{B}^{-1} A \sqrt{B}^{-1}, I d\right), \quad A, B \in S(n),
$$

where $\sqrt{B}$ is the symmetric positive defined square root of $B$. The distance between $A \in S(n)$ and the $I d$ is given by

$$
\delta(A, I d)=\|\log A\|=\left(\sum_{i=1}^{n}\left(\log \lambda_{i}\right)^{2}\right)^{\frac{1}{2}},
$$

where $\lambda_{1}, \ldots, \lambda_{n}$ are the eigenvalues of the matrix $A$.

The following positive answer to the problem of convergence of conformal structures is due to Tukia [29]:

Good Approximation Lemma. Suppose the $K$-quasiregular mappings $f_{j}$ converge to the non-constant mapping $f$. Suppose further that the conformal structures $\mu_{f_{j}}=f_{j}^{*} I d$ converge in measure to $\mu$. Then $\mu=\mu_{f}:=f^{*} I d$.

Proof. It suffices to verify the lemma for the restriction of the mappings to an arbitrary small domain $D$ on which $f$ is injective. Then the $f_{j}$ are also injective provided $j$ is big enough. But for quasiconformal mappings the good approximation lemma is precisely Corollary D of [29].

\section{Conical points}

3.1. Definition of the conical points. The concept of conical limit points has many important applications in the setting of Kleinian groups. Recall that a point $x_{0} \in \mathbb{S}^{n-1}$ is called a conical point of the Kleinian group $\Gamma \subset M \ddot{o} b\left(\mathbb{B}^{n}\right)$ if there are elements $\gamma_{j} \in \Gamma$ and a geodesic ray $\sigma \subset \mathbb{B}^{n}$ ending at $x_{0}$ so that the hyperbolic distance $\delta\left(\gamma_{j}(0), \sigma\right)$ stays bounded and $\gamma_{j}(0) \rightarrow x_{0}$. These conical points are precisely the points that allow a nice renormalization: there is $\rho_{j} \rightarrow 0^{+}$so that

$$
\gamma_{j}^{-1} \circ \alpha_{j}(x)=\gamma_{j}^{-1}\left(\rho_{j} x+x_{0}\right) \rightarrow \Psi(x),
$$

a non-constant conformal map. If we compare this with Zalcman's lemma, then we see that it is natural to consider the points $x_{0}$ of the Julia set of a given map $f$ for which we have a renormalization (2.2) with a constant translation vector $x_{j} \equiv x_{0}$. We use a local version of this to define the set of conical points as in Lemma 1.1. 
Definition 3.1. A point $x_{0}$ is called a conical point for the uqr mapping $f$ if there are a sequence of positive numbers $\rho_{j} \searrow 0$ and a sequence of iterates $f^{k_{j}}$ so that

$$
f^{k_{j}}\left(x_{0}+\rho_{j} x\right) \rightarrow \Psi(x)
$$

uniformly on $\mathbb{B}^{n}$, where $\Psi: \mathbb{B}^{n} \rightarrow \overline{\mathbb{R}}^{n}$ is a non-constant quasimeromorphic mapping. The set of conical points is denoted $\Lambda_{c}$.

From the above discussion (see also the preamble to Lemma 1.1) we see that this definition is universal in the sense that it applies equally to Kleinian groups, rational functions, and also to uqr maps.

3.2. The case of rational maps. A nice feature of the conical set is that it is always a large subset of the Julia set, and in many cases we even have the equality $\Lambda_{c}=\mathcal{J}_{f}$. In particular, this is true for all known uqr maps in dimensions $n \geq 3$. To illustrate what happens in two dimensions, we now discuss in greater detail the notion of conical points for rational functions. First of all we formulate an equivalent definition of $\Lambda_{c}$ :

Lemma 3.2. Let $f$ be a rational function, and denote by $U\left(x_{0}, f^{k}, \delta\right)$ the component of $f^{-k}\left(\mathbb{D}\left(f^{k}\left(x_{0}\right), \delta\right)\right)$ that contains $x_{0}$. The point $x_{0}$ is conical if and only if there are constants $\delta>0, d \in \mathbb{N}$ and a sequence $k_{j} \rightarrow \infty$ so that

$$
f^{k_{j}}: U\left(x_{0}, f^{k_{j}}, \delta\right) \rightarrow \mathbb{D}\left(f^{k_{j}}\left(x_{0}\right), \delta\right)
$$

has degree no more than $d$.

Proof. Clearly, if $x_{0} \in \Lambda_{c}$, then (3.2) holds with $d$ being the degree of the nonconstant limit map at $x_{0}$. Conversely, if (3.2) is true, then we see from the maximum principle that the components $U_{j}=U\left(x_{0}, f^{k_{j}}, \delta\right)$ are simply connected domains. Now choose $\alpha_{j}(x)=\rho_{j} x+x_{0}$ with $\rho_{j}>0$ maximal so that $\alpha_{j}(\mathbb{D}) \subset U_{j}$. Then $\Psi_{j}=f^{k_{j}} \circ \alpha_{j}$ is a normal family, and since the degree of these maps is uniformly bounded it easily follows from the distortion lemma [2, Lemma 2.2] that any limit function of a convergent subsequence of $\left\{\Psi_{j}\right\}$ cannot be a constant map.

Several other notions of conical points appear in the literature. From (3.2) we see that the above definition appears in Appendix 3 of 24], where Przytycki compares different notions of conical points. We also see that $\Lambda_{c}$ is somehow in the spirit of the conical set of Lyubich and Minsky [12. McMullen [21] and independently Urbanski [3] call a point conical if the mappings in (3.2) can be chosen to be conformal. Points satisfying the condition (3.1) also have been used and studied in the recent paper [6] of Haïssinsky.

Recall that a rational map $f$ is

- hyperbolic if there is $\delta>0$ so that for any $x \in \mathcal{J}_{f}$ and any $k \in \mathbb{N}$ the map $f^{k}: U\left(x, f^{k}, \delta\right) \rightarrow \mathbb{D}\left(f^{k}(x), \delta\right)$ is injective,

- semi-hyperbolic if there is $\delta>0$ so that for any $x \in \mathcal{J}_{f}$ and any $k \in \mathbb{N}$ the degree of $f^{k}: U\left(x, f^{k}, \delta\right) \rightarrow \mathbb{D}\left(f^{k}(x), \delta\right)$ is at most $d_{0}$, for some fixed $d_{0}$, and

- topological Collet-Eckmann if there are $\delta>0$ and $N \in \mathbb{N}$ so that for any $x \in \mathcal{J}_{f}$ there is a subsequence $k_{j}$ so that first $k_{j+1} \leq N k_{j}$ and second the degree of $f^{k_{j}}: U\left(x, f^{k_{j}}, \delta\right) \rightarrow \mathbb{D}\left(f^{k_{j}}(x), \delta\right)$ is at most $d_{0}$, with again $d_{0}$ some fixed maximal degree. 
From (3.2) it immediately follows that $\Lambda_{c}=\mathcal{J}_{f}$ in the case of a hyperbolic, semihyperbolic or topological Collet-Eckmann map $f$. Here is another fact illustrating that $\Lambda_{c}$ is a big subset of the Julia set for general maps. It is a consequence of work of Ledrappier (see [4, p.611]).

Theorem 3.3. Let $f$ be a rational function and $\mu$ any $f$-conformal probability measure with positive Lyapunov exponent (for example, $\mu=\mu_{f}$, the maximal entropy measure). Then the conical set has full $\mu$-measure, i.e. $\mu\left(\Lambda_{c}\right)=1$.

3.3. General properties of $\Lambda_{c}$. We finally discuss some elementary properties of the conical set $\Lambda_{c}$. A nice property is the following:

Lemma 3.4. The set of conical points is completely invariant, i.e.

$$
\Lambda_{c}=f\left(\Lambda_{c}\right)=f^{-1}\left(\Lambda_{c}\right) .
$$

Proof. This follows immediately from the standard distortion estimates (see for example [26, p.37]).

From the renormalization description we see that conical points dynamically behave like repelling points. It is no surprise, though, that neutral fixed points cannot be in $\Lambda_{c}$.

Lemma 3.5. Conical points that are also fixed points are always repelling.

Proof. Suppose $x_{0}$ is a fixed point of $f$. If $x_{0} \in \Lambda_{c}$, then there are constants $\delta>0, d \in \mathbb{N}$ and a sequence of components $U_{j}=U\left(x_{0}, f^{n_{j}}, \delta\right)$ with $\overline{U_{j+1}} \subset U_{j}$ and so that $f^{n_{j}}: U_{j} \rightarrow \mathbb{B}\left(x_{0}, \delta\right)$ has degree less or equal to $d$. Observe that $f^{n_{j+1}-n_{j}}: U_{j+1} \rightarrow U_{j}$ and that all but a finite number of these mappings have to be injective. It follows that $x_{0}$ is a repelling fixed point.

In our theorems the condition $\left|\Lambda_{c}\right|>0$ appears. Let us clarify that in fact we have the following dichotomy:

Lemma 3.6. The conical set of any uqr map $f$ has either zero or full Lebesgue measure.

Proof. Suppose that $\left|\Lambda_{c}\right|>0$. It suffices then to renormalize a density point $x_{0} \in \Lambda_{c}$ to find an open set $\Omega$ such that $\Lambda_{c}$ has full measure in $\Omega$. The conclusion follows then from (2.1) since $\mathcal{J}_{f} \cap \Omega \neq \emptyset$.

\section{LATTÈS EXAMPLES}

Since they play an essential role in this paper, we recall here the construction and some properties of the (chaotic) Lattès examples of [17. We refer to this paper for more details.

The construction is based on the existence of quasimeromorphic versions of the Weierstrass $\mathcal{P}$-function (compare [16]). This is a quasimeromorphic map $h: \mathbb{R}^{n} \rightarrow$ $\overline{\mathbb{R}}^{n}$ that is automorphic with respect to a crystallographic group $\Gamma \subset \operatorname{Isom}\left(\mathbb{R}^{n}\right)$, i.e., $h$ has the following two properties:

1) $h \circ \gamma=h$ for all $\gamma \in \Gamma$, and

2) $\Gamma$ acts transitively on fibers: Whenever $h\left(x_{1}\right)=h\left(x_{2}\right)$, there exists an element $\gamma \in \Gamma$ such that $\gamma\left(x_{1}\right)=x_{2}$. 
Due to these properties, the image $\overline{\mathbb{R}}^{n}=h\left(\mathbb{R}^{n}\right)$ can be identified with the orbifold $\mathbb{R}^{n} / \Gamma$. Now, every expanding similarity $A(x)=\lambda U x, \lambda>1$ and $U \in O(n)$, can be projected by $h$ to a uqr map $f$ provided $A$ satisfies the condition $A \circ \Gamma \circ A^{-1} \subset \Gamma$. Then we have the following diagram:

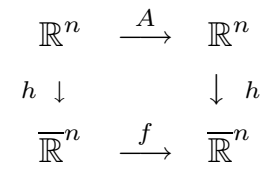

In this way we get a family of uqr maps that all have a chaotic dynamical behavior: Their Julia set is the whole space $\mathcal{J}_{f}=\overline{\mathbb{R}}^{n}$. In two dimensions, with $h$ the holomorphic Weierstrass $\mathcal{P}$-function and with $A(z)=2 z$, the function $f_{\text {Lat }}$ is precisely the function Lattès constructed in order to get the first example of a rational map with $\mathcal{J}_{f}=\hat{\mathbb{C}}$.

It is easy to see that Lattès maps are semi-hyperbolic. Therefore $\Lambda_{c}=\mathcal{J}_{f}=\overline{\mathbb{R}}^{n}$.

For these Lattès maps, one knows precisely all the possible invariant conformal structures (see [17, Thm. 3]). The canonical way to associate an invariant conformal structure $\mu$ to a map $f$ that is defined by (4.1) is to push forward the standard Euclidean structure: $I d=h^{*} \mu$. The special property of Lattès maps is that they may have several invariant conformal structures. In fact, several crystallographic groups $\Gamma$ preserve other (constant) structures $M$ than just the Euclidean $I d$ structure. If $A$ also preserves it, then $M$ induces an $f$-invariant structure $\nu$ which is different from the previous one and given by $M=h^{*} \nu$. In the case of Lattès' original example $f_{\text {Lat }}$ it is easy to check that any constant matrix $M \in S(2)$ gives rise to an invariant conformal structure.

In the case where $h$ is automorphic with respect to a group that is not cocompact, one obtains two other types of examples: Maps that behave like power or Tchebyshev polynomials. This larger class of maps is called Lattès-type maps.

\section{Rigidity on the CONICAL SET OF RATIONAL FUNCTIONS}

We prove here a pointwise rigidity result which implies Theorem 1.2 .

Theorem 5.1 (Pointwise rigidity for rational maps). Let $f$ be a rational function and $\mu$ an $f$-invariant nontrivial dilatation. If $\mu$ is continuous in measure at a conical point $x_{0} \in \Lambda_{c}$, then $f$ is a Lattès map.

Recall that a measurable function $\mu$ is continuous in measure at a point $x_{0}$ if for every $\varepsilon>0$

$$
\lim _{r \rightarrow 0} \frac{1}{\left|\mathbb{D}\left(x_{0}, r\right)\right|}\left|\left\{x \in \mathbb{D}\left(x_{0}, r\right) ; \delta\left(\mu(x), \mu\left(x_{0}\right)\right)>\varepsilon\right\}\right|=0,
$$

and that a measurable function is continuous in measure almost everywhere (see [5. Thm. 2.9.13], where this notion is called approximate continuity).

Theorem 1.2 is a direct consequence of the above result:

Proof of Theorem 1.2. Suppose that the rational map $f$ has an invariant line field. Then it also has a (nontrivial) $f$-invariant conformal structure $\mu$. This function $\mu$ is measurable and hence continuous in measure almost everywhere. Since $\left|\Lambda_{c}\right|>0$, there is $x_{0} \in \Lambda_{c}$ with $\mu$ continuous in measure at $x_{0}$. The result follows now from Theorem 5.1 , 
A further application is that a quasiconformal deformation cannot be smooth at a conical point.

Corollary 5.2. Suppose $f$ and $g$ are rational functions and $\varphi$ a quasiconformal map (but not a Möbius transformation) such that $g \circ \varphi=\varphi \circ f$. If the derivative of $\varphi$ is continuous in measure at a conical point of $f$, then $f$ and $g$ are Lattès maps.

Proof. The dilatation $\mu=\varphi^{*} I d$ is $g$-invariant and continuous in measure at a conical point. The corollary now follows from Theorem 5.1.

There is an analogous result in the context of Kleinian groups due to Ivanov 8]. He also has a rigidity result at so-called horospherical points involving some additional smoothness of the conjugating map $\varphi$. One might introduce the notion of horospherical point in holomorphic dynamics and generalize his results to holomorphic dynamics. We shall address this elsewhere.

Proof of Theorem 5.1. Let $x_{0} \in \Lambda_{c}$ with $\mu$ continuous in measure at $x_{0}$. We first renormalize at $x_{0}$ : There are $n_{j} \rightarrow \infty$ and $\alpha_{j}(x)=r_{j} x+x_{0}$ so that

$$
\Psi=f^{n_{j}} \circ \alpha_{j} \rightarrow \Psi: \mathbb{D} \rightarrow \Omega=\Psi(\mathbb{D})
$$

uniformly with $\Psi$ a non-constant holomorphic map. Consider the pull-back dilatations $\nu=\Psi^{*} \mu$ and $\nu_{j}=\Psi_{j}^{*} \mu$. From the $f$-invariance of $\mu$ it follows that

$$
\nu_{j}=\alpha_{j}^{*}\left[\left(f^{n_{j}}\right)^{*} \mu\right]=\alpha_{j}^{*} \mu=\mu \circ \alpha_{j} .
$$

Let $\varepsilon>0$ and consider the set

$$
\mathcal{E}_{\varepsilon, j}=\left\{x \in \mathbb{D} ; \delta\left(\nu_{j}(x), \mu\left(x_{0}\right)\right)>\varepsilon\right\}=\left\{w \in D_{j}=\alpha_{j}(\mathbb{D}) ; \delta\left(\mu(w), \mu\left(x_{0}\right)\right)>\varepsilon\right\} .
$$

Then, from the continuity in measure of $\mu$ at $x_{0}$, we see that

$$
\left|\mathcal{E}_{\varepsilon, j}\right|=\frac{1}{\left|D_{j}\right|}\left|\left\{w \in D_{j}=\alpha_{j}(\mathbb{D}) ; \delta\left(\mu(w), \mu\left(x_{0}\right)\right)>\varepsilon\right\}\right| \rightarrow 0
$$

when $j \rightarrow \infty$, meaning that $\nu_{j}$ converges in measure to the constant dilatation $\nu \equiv \mu\left(x_{0}\right)$.

We claim that this constant dilatation $\nu \equiv \mu\left(x_{0}\right)$ is non-trivial. In fact, suppose that $\nu \equiv I d$ on $\mathbb{D}$. The map $\Psi$ being holomorphic, this would imply $\mu \equiv I d$ on the domain $\Omega$. Since $\Omega \cap \mathcal{J}_{f} \neq \emptyset$, there is for any $\varepsilon>0$ an integer $n$ so that $f^{n}(\Omega) \supset \hat{\mathbb{C}} \backslash \mathcal{N}_{\varepsilon}\left(\mathcal{E}_{f}\right)$, with $\mathcal{N}_{\varepsilon}\left(\mathcal{E}_{f}\right)$ the $\varepsilon$-neighborhood of the exceptional set. It follows that $\mu \equiv I d$, contradicting our standard hypothesis that $\mu$ is nontrivial.

Now choose a point $w \in \Omega$ of a repelling cycle and suppose that this is a fixed point of $g=f^{k}$. Up to composition of $\Psi$ by a translation we may suppose that $\Psi(0)=w$. In a neighborhood $U$ of this origin we can define $\varphi$ by

$$
\Psi \circ \varphi(x)=g \circ \Psi(x) \text { for } x \in U .
$$

For this holomorphic map $\varphi$, the dilatation $\nu$ is invariant, i.e. $\varphi^{*} \nu=\nu$. Therefore, if $\theta_{x}$ is the direction of the major axis of the ellipse field generated by $\mu$, we have

$$
\theta_{\varphi(x)}=\theta_{x}+\arg \varphi^{\prime}(x) \bmod \pi .
$$

In fact, we shall consider the associated invariant line field. Remember that $\nu$ is constant, and so $\theta_{\varphi(x)}=\theta_{x}$. It follows that

$$
\varphi(x)=\lambda x \quad \text { for some real } \lambda .
$$


From (5.1) we see that $\Psi$ linearizes $g$ at $w$, and this map can be extended in the usual way using (5.1) to a global map $\Psi: \mathbb{C} \rightarrow \hat{\mathbb{C}} \backslash \mathcal{E}_{f}$ such that $g \circ \Psi(x)=\Psi \lambda(x)$ for every $x \in \mathbb{C}$.

We finally verify that $\Psi$ is in fact an elliptic function. Consider two points $x_{1}$ and $x_{2}$ (neither one critical) with $\Psi\left(x_{1}\right)=\Psi\left(x_{2}\right)$. Then one can define near $x_{1}$ a map $\gamma$ with $\Psi \circ \gamma=\Psi$. This holomorphic map $\gamma$ also preserves $\nu$, and, as before, it follows that $\gamma(x)=\alpha x+\beta$ (with $\alpha$ real). The set of all possible such mappings

$$
\Gamma=\{\gamma \text { affine with } \Psi \circ \gamma=\Psi\}
$$

is a discrete group that acts transitively on fibers of $\Psi$. Consequently, the elements of $\Gamma$ have to be isometries, and $g$ is a power, Tchebyshev or Lattès map. Moreover, since power and Tchebyshev maps are (globally) rigid, $g$ has to be a Lattès map.

It then follows that $f$ itself is a Lattès map. For example, using again the invariant line field argument (5.2), one sees that $\Psi$ linearizes $f$ at any (necessarily repelling) fixed point, and hence the automorphic function $\Psi$ semiconjugates a scalar multiplication and $f$.

\section{Rigidity OF UNIFORMLY QUASIREGULAR MAPPINGS}

In this section we show that the chaotic Lattès maps are the only uqr mappings with a set of conical points of positive measure.

Theorem 6.1 (Pointwise rigidity for uqr maps). Suppose $f$ is a $\mu$-rational map of $\overline{\mathbb{R}}^{n}, n \geq 3$, and that $\mu$ is continuous in measure at a conical point $x_{0} \in \Lambda_{c}$. Then $f$ is a Lattès-type map.

As a first consequence we have the following.

Proof of Theorem 1.3. Since a measurable function is almost everywhere continuous in measure, Theorem 1.3 follows immediately from Theorem 6.1

There is another rigidity phenomenon which follows from Theorem 6.1; For a hyperbolic Blaschke product $B$ of the unit disc, one knows that the induced circle action $B: \mathbb{S}^{1} \rightarrow \mathbb{S}^{1}$ is quasisymmetrically conjugate to $z \mapsto z^{d}$, where $d$ is the degree of $B$. But this conjugacy is always singular, except when $B$ is actually the power map $z \mapsto z^{d}$ (see [28]). In higher dimensions we also have natural uqr power maps [17. Their Julia (and conical) set is the sphere $\mathbb{S}^{n-1}$, and their restrictions to this sphere are Lattès maps. Theorem 6.1 implies that all higher-dimensional "hyperbolic uqr-Blaschke products" share this property:

Corollary 6.2. Let $f$ be a uqr map of $\overline{\mathbb{R}}^{n}$, let $n \geq 4$ and suppose that $\Lambda_{c}=\mathcal{J}_{f}=$ $\mathbb{S}^{n-1}$. Then the restriction of $f$ to $\mathbb{S}^{n-1}$ is a Lattès map.

There is one dimension left, and it is natural to ask what happens when $n=3$. This problem leads to the question of which chaotic rational maps do admit a uqr extension to the 3-ball. Lattès maps have such an extension, namely the power mappings of [17]. The above corollary suggests that these are the only exceptions.

Proof of Theorem 6.1. We start with the same renormalization argument as in the Proof of Theorem 5.1. Since $x_{0} \in \Lambda_{c}$, there are $\rho_{j} \rightarrow 0^{+}$and $k_{j} \rightarrow \infty$ so that

$$
\Psi_{j}(x)=f^{k_{j}} \circ \alpha_{j}(x)=f^{k_{j}}\left(\rho_{j} x+x_{0}\right) \rightarrow \Psi(x), \quad x \in \mathbb{B}^{n},
$$


uniformly on $\mathbb{B}^{n}$, where $\Psi$ is non-constant and quasimeromorphic. Consider again the pull-back structures $\nu=\Psi^{*} \mu$ and $\nu_{j}=\Psi_{j}^{*} \mu$. From the $f$-invariance of $\mu$ and the fact that $\mu$ is continuous in measure at $x_{0}$, it follows, precisely as in the proof of Theorem 5.1, that $\nu_{j}$ converges to the constant structure $x \mapsto \mu\left(x_{0}\right)$. The good approximation lemma asserts that

$$
\nu=\lim _{j \rightarrow \infty} \nu_{j} \equiv \mu\left(x_{0}\right) .
$$

Now choose $L: \mathbb{R}^{n} \rightarrow \mathbb{R}^{n}$ affine with $L^{*} \mu\left(x_{0}\right)=I d$, and denote

$$
h=\Psi \circ L: \mathbb{B}^{n} \rightarrow \Omega=h\left(\mathbb{B}^{n}\right) .
$$

Then $h^{*} \mu:=(\Psi \circ L)^{*} \mu=I d$.

The next step is to lift some iterate of $f$ by $h$. Let $V=\mathbb{B}(h(0), \delta) \subset \Omega$, and $U$ the connected component of $h^{-1}(V)$ that contains 0 . If $\delta$ is sufficiently small, then $h: U \rightarrow V$ is a proper map (see [26]). Since

$$
\Psi_{j+N}(x)=f^{k_{j+N}-k_{j}} \circ \Psi_{j}\left(\frac{\rho_{j+N}}{\rho_{j}} x\right),
$$

we can choose $l=k_{j+N}-k_{j}>>0$ so that $\overline{V_{0}} \subset V$, where $V_{0}$ is the component of $f^{-l}(V)$ that contains $\Psi_{j}(0)$. Denote finally by $U_{0} \subset U$ a component of $h^{-1}\left(V_{0}\right)$. We therefore have the following diagram of proper mappings:

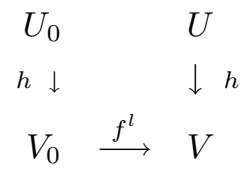

In this picture there is one map missing, say $A: U_{0} \rightarrow U$. We obtain it in lifting $f^{l}$. In order to do this, let $W \subset V$ be a domain such that a branch $H: W \rightarrow W^{\prime}=$ $H(W) \subset U$ of $h^{-1}$ exists, and denote by $W_{0}^{\prime} \subset U_{0}$ a component of $\left(f^{l} \circ h\right)^{-1}(W)$. Then we can define

$$
A:=H \circ f^{l} \circ h: W_{0}^{\prime} \rightarrow W^{\prime} .
$$

Since the $h$-pull-back of $\mu$ is the standard structure and $\mu$ is $f$-invariant, the lifted map $A$ has the property $A^{*} I d=I d$.

From Liouville's theorem it follows that $A$ is (the restriction of) a Möbius transformation. Using analytic continuation (cf. the proof of Proposition 6.3 in [18]), we see that

$$
h \circ A(x)=f^{l} \circ h(x) \quad \text { for every } x \in U_{0} .
$$

Since $\overline{U_{0}} \subset U$, the map $A$ is a loxodromic Möbius transformation with a repelling fixed point $a_{0} \in U_{0}$. We may suppose that the repelling fixed point is $\infty$.

The map $h$ linearizes $f^{l}$ at the repelling fixed point $b=h(a)$ and, using (6.2), we can extend this map to $h: \mathbb{R}^{n} \rightarrow \overline{\mathbb{R}}^{n}$ so that the conjugation (6.2) is true globally.

At this stage we can conclude using [18, §6]. Let us just briefly outline how to proceed. Suppose that $x_{1}, x_{2}$ are two points that are not branch points of $h$ and so that $h\left(x_{1}\right)=h\left(x_{2}\right)=y$. Then we can define locally an inverse branch $H$ of $h$ with $H(y)=x_{2}$. This defines locally near $x_{1}$ a map $\gamma=H \circ h$ sending $x_{1}$ onto $x_{2}$. Clearly $\gamma$ is an $I d$-rational map, and hence the restriction of a Möbius transformation. Using analytic continuation again, we see that $h \circ \gamma=h$ is true in the whole of $\mathbb{R}^{n}$. It suffices now to consider the set

$$
\Gamma=\left\{\gamma \in M \ddot{o} b\left(\mathbb{R}^{n}\right) ; h \circ \gamma=h\right\} .
$$


It turns out that $\Gamma$ is a discrete group of isometries and that $h$ is automorphic with respect to this group.

We have shown that $f^{l}$ is of Lattès-type, and therefore so is $f$ (see [18, Prop. $5.1])$.

\section{REFERENCES}

[1] F. Berteloot and J.-J. Loeb, Spherical Hypersurfaces and Lattès Rational Maps, J. Math. Pures Appl. 77 (1998), 655-666. MR 99e:32043

[2] L. Carleson, P.W. Jones, and J.-Ch. Yoccoz, Julia and John, Bol. Soc. Bras. Mat. 25 (1994), 1-30. MR 95d:30040

[3] M. Denker, R.D. Mauldin, Z. Nitecki, and M. Urbanski, Conformal measures for rational functions revisited, Fund. Math. 157 (1998), 161-173. MR 99j:58122

[4] A.E. Eremenko and M. Yu. Lyubich, The dynamics of analytic transformations, Leningrad Math. J. 1 (1990), 563-634. MR 91b:58109

[5] H. Federer, Geometric Measure Theory, Springer Verlag (1969). MR 41:1976

[6] P. Haïssinsky, Rigidity and expansion for rational maps, J. London Math. Soc. (2) 63 (2001), 128-140. MR 2001m:37085

[7] S. Helgason, Differential Geometry, Lie Groups and Symmetric Spaces, Academic Press, 1978. MR 80k:53081

[8] N. I. Ivanov, Action of Möbius Transformations on Homeomorphisms: Stability and Rigidity, Geom. Funct. Anal. 6 (1996), 79-119. MR 97e:30075

[9] T. Iwaniec and G.J. Martin, Quasiregular Semigroups, Ann. Acad. Sci. Fenn. Math. 21 (1996), 241-254. MR 97i:30032

[10] O. Lehto and K.I. Virtanen, Quasiconformal mappings in the plane, Springer-Verlag, 1973. MR 49:9202

[11] M. Lyubich, The dynamics of rational transforms: the topological picture, Russian Math. Surveys 41 : 4 (1986), no. 4, 43-117. MR 88g:58094

[12] M. Lyubich and Y. Minsky, Laminations in holomorphic dynamics, J. Diff. Geometry 47 (1997), 17-94. MR 98k:58191

[13] R. Mañé, P. Sad and D. Sullivan, On the dynamics of rational maps, Ann. Scient. Éc. Norm. Sup. (4) 16 (1983), 193-217. MR 85j:58089

[14] G.J. Martin, Branch sets of uniformly quasiregular maps, Conformal Geometry and Dynamics 1 (1997), 24-27. MR 98d:30032

[15] G.J. Martin and V. Mayer, Local Dynamics of Uniformly Quasiregular mappings, preprint.

[16] O. Martio and U. Srebro, Periodic Quasimeromorphic Mappings in $\mathbb{R}^{n}$, J. d'Analyse Math. 28 (1975), 20-40.

[17] V. Mayer, Uniformly Quasiregular mappings of Lattès' type, Conformal Geometry and Dynamics 1 (1997), 104-111. MR 98j:30017

[18] V. Mayer, Quasiregular analogues of critically finite rational functions with parabolic orbifold, J. d'Analyse Math. 75 (1998), 105-119. MR 2000a:30043

[19] C. McMullen, Area and Hausdorff dimension of Julia sets of entire functions, Trans. Amer. Math. Soc. 300 (1987), 329-342. MR 88a:30057

[20] C. McMullen, Complex Dynamics and Renormalization, Ann. Math. Studies, Princeton University Press (1994). MR 96b:58097

[21] C. McMullen, Hausdorff dimensions and conformal dynamics II: Geometrically finite rational maps, Comment. Math. Helv. 75 (2000), 535-593. MR 2001m:37089

[22] C. McMullen and D. Sullivan, Quasiconformal Homeomorphisms and Dynamics III. The Teichmüller Space of a Holomorphic Dynamical System, Adv. in Math. 135 (1998), 351395. MR 99e:58145

[23] R. Miniowitz, Normal families of quasimeromorphic mappings, Proc. AMS 84 (1982), 3543. MR 83c:30026

[24] F. Przytycki, Conical limit set and Poincaré exponent for iterations of rational functions, Trans. Amer. Math. Soc. 351 (1999), 2081-2099. MR 99h:58110

[25] M. Rees, Positive measure sets of ergodic rational maps, Ann. Scient. Éc. Norm. Sup. 19 (1986), 383-407. MR 88g:58100

[26] S. Rickman, Quasiregular Mappings, Springer-Verlag (1993). MR 95g:30026

[27] J.L. Schiff, Normal families, Universitext Springer-Verlag (1993). MR 94f:30046 
[28] M. Shub and D. Sullivan, Expanding endomorphisms of the circle revisited, Ergodic Theory and Dynamical Systems 5 (1985), 285-289. MR 87g:58104

[29] P. Tukia, On quasiconformal groups, J. d'Analyse Math. 46 (1986), 318-346. MR 87 m:30043

[30] L. Zalcman, A heuristic principle in complex function theory, Amer. Math. Monthly 82 (1975), 813-817. MR 52:757

Department of Mathematics, University of Auckland, Auckland, New Zealand

E-mail address: martin@math.auckland.ac.nz

UMR 8524 du CNRS - UFR de Mathématiques, Université des Sciences et Technologies de Lille, 59655 Villeneuve d'AscQ Cedex, France

E-mail address: volker.mayer@univ-lille1.fr 